\title{
A Practical Guide to Analyzing and Reporting the Movement of Nanoscale Swimmers
}

Wei Wang ${ }^{1 *}$ and Thomas E. Mallouk ${ }^{2 *}$

1. School of Materials Science and Engineering, Harbin Institute of Technology, Shenzhen, China

2. Department of Chemistry, University of Pennsylvania, Philadelphia, PA, USA

weiwangsz@hit.edu.cn mallouk@sas.upenn.edu

\section{Description for MATLAB codes}

Two zip files are uploaded as Supporting Information, which serve the purpose of correcting drift from a recorded video, and overlaying speed/time information on a video.

The folder "Drift_correction" contains a .txt file "sample_data_with_drift", which can be used as a practice set. This data set had been produced by tracking the "sample_video.avi" file in the folder "Plotting_trajectories". In this video, $5 \mu \mathrm{m}$ polystyrene beads are suspended in water, and an arbitrary drift toward top left was introduced by blowing wind to the suspension. The four columns in the txt file are the $\mathrm{X}$ coordinate (in pixels), $\mathrm{Y}$ coordinate (in pixels), frame number and the track number, respectively. After loading this folder in MATLAB, load the "use_this_for_drift_correction.m" file and run it. A plot with four curves will be generated that show the calculated drift along the $\mathrm{x}$ (red solid line) and $\mathrm{y}$ (blue solid line). These two curves are fitted, shown in dashed lines. Often, the presence of drift becomes obvious by examining the magnitude of the calculated drift. A drift of a few pixels over tens of seconds is probably insignificant. Drift much larger than that, as shown in this example, cannot be ignored and must be corrected. In addition to this plot, a .txt and a .xml file carrying the name of "sample_data_with_drift_correct" are generated, which are the data after drift correction, and can then be used for plotting trajectories and calculating MSD, as we show in Fig. 4 in the main text. Comments in the code provide guidance if modifications of the code become necessary.

Codes in the folder "Plotting_trajectories" are used to overlay a recorded video of nanoswimmers with their instantaneous speeds or elapsed time. This folder also contains a sample .txt file that can be used in the same way as above. The data from this sample.txt file had been generated from the provided video file sample_video.avi, upon which the speeds or time are overlaid in this tutorial. The key parameter that needs attention is the $\operatorname{tr}(\mathrm{ttt}, 3)$ in line 53. By default, the " 3 " here means the video will be overlaid with the speeds of each swimmer. In case elapsed time is preferred, change " 3 " to " 5 ". The "track number", i.e. the red number on the top right of each swimmer, can be turned off by bypassing line 57-63. The overlaid video can be viewed in real time as it is generated, and this procedure can take some time if a long video is being processed. Upon completion, this code generates a new video file starting with "Overlaid_". 